\title{
Emergency Department Care for Patients with Limited English Proficiency: a Retrospective Cohort Study
}

\author{
Lucy Schulson, $M D, M P H^{7}$, Victor Novack, $M D, P h D^{2,3}$, Peter B. Smulowitz, $M D^{4}$, Tenzin Dechen, $M P H^{2}$, \\ and Bruce E. Landon, M.D., M.B.A., M.Sc. ${ }^{7,2,5}$
}

'Division of General Medicine and Primary Care, Department of Medicine, Beth Israel Deaconess Medical Center, Boston, MA, USA; ${ }^{2}$ Center for Healthcare Delivery Science, Beth Israel Deaconess Medical Center, Boston, MA, USA; ${ }^{3}$ Clinical Research Center, Faculty of Health Sciences, Soroka University Medical Center, Ben-Gurion University of the Negev, Beersheba, Israel; ${ }^{4}$ Department of Emergency Medicine, Beth Israel Deaconess Medical Center, Boston, MA, USA; ${ }^{5}$ Department of Health Care Policy, Harvard Medical School, Boston, MA, USA.

BACKGROUND: Limited English proficiency (LEP) patients may be particularly vulnerable in the high acuity and fast-paced setting of the emergency department (ED). OBJECTIVE: To compare the care processes of LEP patients in the ED.

DESIGN: Retrospective cohort study.

SETTING: ED in a large tertiary care academic medical center.

PATIENTS: Adult LEP and English Proficient (EP) patients during their index presentation to the ED from September 1, 2013, to August 31, 2015. LEP patients were identified as those who selected a preferred language other than English when registering for care.

MAIN MEASURES: Rates of diagnostic studies, admission, and return visits for those originally discharged from the ED.

KEY RESULTS: We studied 57,435 visits of which 5241 (9.1\%) were for patients with LEP. In adjusted analyses, LEP patients were more likely to receive an X-ray/ultrasound (OR 1.11, CI 1.03-1.19) and be admitted to the hospital (OR 1.09, CI 1.01-1.19). There was no difference in 72-h return visits (OR 0.98, CI 0.73-1.33). LEP patients presenting with complaints related to the cardiovascular system were more likely to receive a stress test (OR 1.51, CI 1.22-1.86), and those with gastrointestinal diagnoses were more likely to have an X-ray/ultrasound (OR 1.31, CI 1.02-1.68). In stratified analyses, Spanish speakers were less likely to be admitted (OR 0.8, CI 0.70-0.91), but those preferring "other" languages, which were all languages with $<500$ patients, had a statistically significant higher adjusted rate of admission (OR 1.35, CI 1.17-1.57). CONCLUSIONS: ED patients with LEP experienced both increased rates of diagnostic testing and of hospital admission. Research is needed to examine why these differences occurred and if they represent inefficiencies in care.

KEY WORDS: limited English proficiency; emergency room; health disparities

The abstract of this paper was presented as an oral presentation at the Society of General Internal Medicine National meeting on April 22, 2016.

Electronic supplementary material The online version of this article (https://doi.org/10.1007/s11606-018-4493-8) contains supplementary material, which is available to authorized users.

Received February 5, 2018

Revised March 30, 2018

Accepted May 11, 2018

Published online September 5, 2018
J Gen Intern Med 33(12):2113-9

DOI: $10.1007 / \mathrm{s} 11606-018-4493-8$

(c) Society of General Internal Medicine 2018

\section{INTRODUCTION}

Poor communication between patients and physicians has been associated with worse outcomes and more adverse events. ${ }^{1}-3$ Patients with limited English proficiency (LEP), who make up about $9 \%$ of people in the USA, may be particularly prone to experience communication failures, which might put them at risk for worse health outcomes. ${ }^{4}$ Studies have demonstrated that in the community setting, patients with LEP have lower use of preventive services and poorer health outcomes as compared to English proficient patients (EP). ${ }^{5}-12$ They also report worse access to health care providers and lower satisfaction with their health care. ${ }^{13}-15$ Although these disparities are multifactorial-LEP patients are more likely to be poor, less educated, and lack health insurance-language barriers likely contribute to these disparities. ${ }^{5}$ Moreover, interpreters are inconsistently used in treating patients with LEP, and LEP patients may have more difficulty understanding medical information, even when interpreters are present. ${ }^{16}, 17$

LEP patients may be particularly vulnerable in the emergency department (ED) where the higher acuity and faster paced setting might place patients and providers at higher risk for communication errors. The ED also may have more limited access to professional interpreters because of the unscheduled nature of visits, which makes it difficult to anticipate the need for specific interpreters ahead of time. Real and perceived time pressure may make providers less inclined to use interpreters. ${ }^{18}$ Additionally, even when interpreters are present, the fastpaced environment requires providers to make quick decisions with limited information, which likely is exacerbated for patients whose primary language is discordant from their providers. This may lead to increased diagnostic uncertainty, which, in turn, could lead to differences in care processes and provider decision-making. The environment also may be prone to missed diagnoses or communication errors at time of discharge resulting in increased 72 -h return visits. ${ }^{19}-21$ 
Limited research has examined the processes and outcomes of care of LEP adults presenting to the ED. ${ }^{20}, 22-25$ We therefore sought to study the utilization of testing and outcomes of LEP patients at a large tertiary care academic medical center ED. We hypothesized that LEP patients would receive more diagnostic studies for similar complaints, be more likely to be admitted to the hospital, and, for those discharged from the ED, be more likely to return for an unscheduled visit within $72-\mathrm{h}$.

\section{METHODS}

We conducted a retrospective cohort study over the time period September 1, 2013, to August 31, 2015, of all patients presenting to the Emergency Department at Beth Israel Deaconess Medical Center (BIDMC), a tertiary care academic medical center with over 50,000 ED visits per year. Data were obtained from administrative data collected at registration and as part of routine patient care. The study was approved by the Institutional Review Board at BIDMC.

\section{Study Sample}

We included the first ED visit for all patients $\geq 18$ years of age who presented for emergency care at BIDMC over the 2-year study period. Patients whose primary ED diagnosis was pregnancy related or who had a primary substance abuse or psychiatric diagnosis were excluded as were patients who died in the ED, left prior to being seen or against medical advice, or refused treatment $(n=4520)$. Similar to prior studies, we also excluded a small number of high utilizing patients with $\geq$ five visits/year $(n=129)$ because care received by this more familiar population might not be reflective of care for LEP patients overall and could lead to biased results. ${ }^{20}, 26$

\section{Identifying Limited English Proficiency}

When initially registering at the hospital, patients are asked their preferred language, and this is recorded in the medical record and used to determine if a patient requires an interpreter for their care. We categorized patients as LEP if a language other than English was their preferred language. For the LEP population, we further classified them according to the most common preferred languages (all with at least 500 visits) including Spanish, Chinese (Mandarin and Cantonese), Cape Verdean, or Russian. All other languages, of which there were over 60 , were categorized as "other."

\section{Outcomes of Interest}

The primary outcomes of interest were use of diagnostic tests ordered in the ED and whether the patient was admitted to the hospital. We examined use of X-ray and/or ultrasound (U/S), computed tomography (CT) and/or magnetic resonance imaging (MRI), electrocardiograms (EKGs), and stress tests (exercise, imaging, or nuclear).
We counted as admissions all patients who were admitted to the hospital or transferred to another hospital, whether under full admission or observation status. We also examined $72 \mathrm{~h}$ unplanned return visits to the ED, which we defined as any patient who returned to the ED for anything other than a scheduled revisit (V76 (follow-up examination) or V04 (need for prophylactic vaccine)) within 72 -h after their index presentation. $^{20}, 27$

\section{Control Variables}

We ascertained age, sex, race, education, marital status, and insurance type from the hospital registration system. Insurance status was classified as commercial, Medicaid/safety net/selfpay, or Medicare. Triage score and time of presentation were obtained from the ED data system. The triage scores are standardized scores assigned by a triage nurse based on the validated Emergency Severity Index Score, which use a 1-5 scale with 1 indicating higher acuity. ${ }^{28}$ Time of visit was coded as either day (defined as 8 a.m. to 6 p.m.) or night (6 p.m. to 8 a.m.).

\section{Classifying Diagnoses and Symptoms}

We classified the primary ED diagnosis into categories based on clinical classification software (CCS) for ICD-9 CM (2015), which groups diagnoses according to organ system. Based on review by one of the authors (LS), we also identified chief complaints related to "chest pain" or "abdominal pain" from the 12,637 unique verbatim chief complaints collected from patients. These complaints can be present with multiple diagnoses with varying levels of acuity and therefore are associated with more clinical discretion and might be more likely to demonstrate differences in testing and admission. ${ }^{25}$ Complaints for which there was uncertainty were resolved by consensus.

\section{Statistical Analyses}

We performed bivariate analyses using $t$ tests or chi-square tests as appropriate. We then estimated multivariable logistic models for each of the outcomes of interest after adjusting for age, race, sex, triage level, type of insurance, time of day of initial presentation to ED, and ICD-9 diagnosis category. We also conducted additional pre-specified sub-analyses by CCS diagnosis category including examining diagnostic testing relevant to specific diagnoses (i.e., disease of the circulatory system and stress test). We also analyzed admission rates by diagnosis category. Because the diagnosis code that is used to determine diagnosis category is determined at the end of the visit and may be influenced by the diagnostic testing ordered, we performed sensitivity analyses looking at two chief complaints and outcomes relevant to those complaints (e.g., EKG, stress test for chest pain, and X-ray/U/S and CT/MRI for abdominal pain). Finally, we performed additional subanalyses stratified by the most common preferred languages: Spanish, Chinese (Mandarin, Cantonese), Cape Verdean, or 
Russian. We calculated odds ratios (ORs) with $95 \%$ confidence intervals (CI). All statistical tests were two-tailed and $\alpha$ was set at 0.05. All analyses were performed using SAS (version 9.4).

\section{RESULTS}

There were 111,176 visits during our time period of which 60,989 were initial visits. Of these initial visits, 57,435 met our inclusion criteria and $5241(9.1 \%)$ were for patients with LEP. When compared to EP patients, LEP patients were more likely to be female ( 56.6 vs. $53.9 \%, p<.0002$ ), older (median age was 59.5 for LEP, STD 19.2 vs. 49.8 for EP, STD 20.7, $p<.0001$ ), married (49.1 vs. $36.5 \%, p<.0001$ ), to visit the ED during daytime hours $(66.2$ vs. $58.6 \%, p<.0001)$, and to be classified as Medicaid or self-pay insurance coverage (41.4 vs. $15.3 \%, p<.0001$ ) (Table 1 ). The triage acuity level at presentation did not differ between the two groups (mean triage score was 3.4 for LEP, STD \pm 0.7 vs. 3.4 for EP, STD $\pm 0.8, p<0.34)$. LEP patients were more likely to have a diagnosis category that was a "disease of the musculoskeletal and connective tissue system" (10.8 vs. $8.7 \%, p<.0001)$ and less likely to have a diagnosis category of "injury and poisoning diagnosis" (16.5 vs. $23.6 \%, p<.0001)$.

\section{Overall Analyses}

In unadjusted analyses (Table 2), LEP patients received more diagnostic testing including X-rays/U/S (41.6 vs. $38.1 \%$, $p<.0001)$, CT/MRI (30.7 vs. $27.3 \%, p<.0001)$, EKGs $(53.3$ vs. $45.7 \%, p<.0001)$, and stress tests ( 4.3 vs. $2.9 \%, p<.001)$. LEP patients were also admitted at higher rates than EP patients $(39.4$ vs. $34.4 \%, p<.0001)$, but there was no difference in 72 -h revisits ( 1.8 vs. $1.6 \%, p=0.17)$. After adjustment, LEP patients remained more likely to receive an X-ray/U/ S (OR 1.11, CI 1.03-1.19) and to be admitted to the hospital (OR 1.09, CI 1.01-1.19).

\section{Stratified Analysis by Diagnosis}

Among patients with a discharge diagnosis of "disease of the circulatory system," in unadjusted analyses, LEP patients were more likely to receive a stress test (OR 1.41, CI 1.181.69), but there was no statically significant difference in receipt of EKGs (OR 1.28, CI 0.96-1.71) or admission (OR 1.12, CI 0.97-1.30) (Table 3). After adjustment, LEP patients remained more likely to receive a stress test (OR 1.51, CI 1.22-1.86). In contrast to the overall results, these patients were less likely to be admitted as compared to EP patients (OR 0.78 , CI 0.65-0.94). Sensitivity analysis based on those presenting with a chief complaint of chest pain demonstrated similar results (online appendix).

Among patients with a discharge diagnosis related to "diseases of the digestive system," LEP patients were more likely to receive an X-ray/U/S (OR 1.37, CI 1.10-1.70), but
Table 1 Characteristics of Study Participants

\begin{tabular}{|c|c|c|c|}
\hline & \multirow{2}{*}{$\begin{array}{l}\begin{array}{l}\text { English } \\
\text { proficient }\end{array} \\
N=52,194\end{array}$} & \multirow{2}{*}{$\begin{array}{l}\text { Limited } \\
\text { English } \\
\text { proficient }\end{array}$} & \multirow[t]{3}{*}{$\begin{array}{l}p \\
\text { value* }\end{array}$} \\
\hline & & & \\
\hline & $\%$ & $\%$ & \\
\hline \multicolumn{3}{|l|}{ Sex } & .0002 \\
\hline Male & 46.1 & 43.4 & \\
\hline Female & 53.9 & 56.6 & \\
\hline \multicolumn{3}{|l|}{ Age group } & $<.0001$ \\
\hline $18-39$ years & 36.1 & 18.2 & \\
\hline $40-49$ years & 13.2 & 12.8 & \\
\hline $50-59$ years & 16.4 & 17.3 & \\
\hline $60-69$ years & 14.9 & 17.5 & \\
\hline$>70$ years & 19.4 & 34.2 & \\
\hline \multicolumn{3}{|l|}{ Marital status } & $<.0001$ \\
\hline Other & 63.5 & 50.9 & \\
\hline Married/life partner & 36.5 & 49.1 & \\
\hline \multicolumn{3}{|l|}{ Race } & $<.0001$ \\
\hline White & 65.7 & 19.9 & \\
\hline Black & 19.1 & 14.9 & \\
\hline Others & 15.3 & 65.2 & \\
\hline \multicolumn{3}{|l|}{ Education } & $<.0001$ \\
\hline Less than high school & 5.4 & 37.5 & \\
\hline High school graduate & 28.9 & 33.6 & \\
\hline College and beyond & 59.7 & 22.7 & \\
\hline $\begin{array}{l}\text { Did not answer/ } \\
\text { unavailable }\end{array}$ & 5.9 & 6.1 & \\
\hline \multicolumn{3}{|l|}{ Payor } & $<.0001$ \\
\hline Commercial & 59.1 & 23.4 & \\
\hline Medicaid/safety net/self- & 15.3 & 41.4 & \\
\hline \multicolumn{4}{|l|}{ pay . } \\
\hline Medicare & 25.5 & 35.2 & \\
\hline \multicolumn{3}{|l|}{ Time of visit } & $<.0001$ \\
\hline Day & 58.6 & 66.2 & \\
\hline Night & 41.3 & 33.7 & \\
\hline \multirow{2}{*}{\multicolumn{3}{|c|}{ Acuity (Emergency }} & \\
\hline & & & .0013 \\
\hline \multicolumn{4}{|l|}{ Severity Index Score $) \dagger$} \\
\hline $1-2$ & 0.2 & 0.2 & \\
\hline 3 & 8.4 & 7.0 & \\
\hline 4 & 53.6 & 55.6 & \\
\hline 5 & 37.8 & 37.2 & \\
\hline \multicolumn{3}{|l|}{ Disease classification } & $<.0001$ \\
\hline Diseases of the circulatory & 14.6 & 16.2 & \\
\hline \multicolumn{4}{|l|}{ system } \\
\hline Injury and poisoning & 23.6 & 16.5 & \\
\hline & 8.7 & 10.8 & \\
\hline \multicolumn{4}{|l|}{$\begin{array}{l}\text { musculoskeletal system } \\
\text { and connective tissue }\end{array}$} \\
\hline Diseases of the nervous & 8.3 & 8.6 & \\
\hline \multicolumn{4}{|l|}{ system and sensory organs } \\
\hline Diseases of the digestive & 8.5 & 7.8 & \\
\hline system & & & \\
\hline Diseases of the respiratory & 7.3 & 8.3 & \\
\hline system & & & \\
\hline $\begin{array}{l}\text { Diseases of the } \\
\text { genitourinary system }\end{array}$ & 5.4 & 7.1 & \\
\hline $\begin{array}{l}\text { Symptoms, signs, and ill- } \\
\text { defined conditions and } \\
\text { factors influencing health } \\
\text { status }\end{array}$ & 11.6 & 11.7 & \\
\hline Others & 11.9 & 12.8 & \\
\hline Languages & & & \\
\hline Spanish & $\mathrm{N} / \mathrm{A}$ & 38.7 & \\
\hline Chinese & $\mathrm{N} / \mathrm{A}$ & 14.5 & \\
\hline Cape Verdean & $\mathrm{N} / \mathrm{A}$ & 12.6 & \\
\hline Russian & $\mathrm{N} / \mathrm{A}$ & 12.0 & \\
\hline Other & $\mathrm{N} / \mathrm{A}$ & 22.1 & \\
\hline
\end{tabular}

$* p<.05$

†Acuity with greatest acuity 1 to least acute being 5 
Table 2 Unadjusted and Adjusted Comparisons of Outcomes for Limited English Proficient vs. English Proficient Patients

\begin{tabular}{|c|c|c|c|c|c|c|c|}
\hline & \multicolumn{3}{|l|}{ Unadjusted percentage $\dagger$} & \multicolumn{2}{|c|}{ Unadjusted OR } & \multicolumn{2}{|c|}{ Adjusted OR: } \\
\hline & Limited English proficient & English proficient & $p$ value* & OR & $95 \% \mathrm{CI}$ & OR & $95 \% \mathrm{CI}$ \\
\hline \multicolumn{8}{|l|}{ Testing and imaging } \\
\hline $\mathrm{X}-\mathrm{ray} / \mathrm{U} / \mathrm{S}$ & 41.6 & 38.1 & $<.0001$ & 1.15 & $1.09-1.22$ & 1.11 & $1.03-1.19$ \\
\hline CT/MRI & 30.7 & 27.3 & $<.0001$ & 1.18 & $1.11-1.26$ & 0.99 & $0.92-1.08$ \\
\hline EKG & 53.3 & 45.7 & $<.0001$ & 1.36 & $1.28-1.44$ & 1.03 & $0.95-1.12$ \\
\hline Stress test & 4.3 & 2.9 & $<.0001$ & 1.45 & $1.25-1.67$ & 1.19 & $0.98-1.44$ \\
\hline \multicolumn{8}{|l|}{ Disposition and outcomes } \\
\hline Admission & 39.4 & 34.4 & $<.0001$ & 1.24 & $1.17-1.32$ & 1.09 & $1.01-1.19$ \\
\hline 72-h unscheduled return visit & 1.8 & 1.6 & 0.17 & 1.00 & $0.78-1.28$ & 0.98 & $0.73-1.33$ \\
\hline
\end{tabular}

CT computed tomography, CI confidence interval, EKG electrocardiogram, MRI magnetic resonance imaging, OR odds ratio, U/S ultrasound *Comparisons with $p<.05$

FPresented as percentage of total of LEP or EP population

$\ddagger$ Odds ratios with EP as reference. Models are adjusted for age, sex, race, marital status education, insurance type, time of presentation, and triage score

$* p<.05$

†Acuity with greatest acuity 1 to least acute being 5

there was no statistically significant difference in receipt of CT/MRI (OR 1.07, CI 0.87-1.33) or in admission (OR 1.04, $0.84-1.29)$. Adjusted analyses were similar. In sensitivity analyses examining patients presenting with a gastrointestinal chief complaint, results were consistent although the use of $\mathrm{X}$ $\mathrm{ray} / \mathrm{U} / \mathrm{S}$ was no longer statistically significant (OR 1.20 , CI $0.95-1.51)$. Given the low rates of 72 -h revisits, this outcome was not included in sub-analyses.

\section{Stratified Analyses by Primary Language (Table 4)}

In adjusted analyses, Spanish speakers were statistically significantly more likely than EP patients to have an X-ray/U/S (OR 1.20, CI 1.08-1.34), CT/MRI (OR 1.43 CI 1.34-1.54), and stress test (OR 1.65, CI 1.24-2.21) as compared to EP patients. Chinese and Cape Verdean speakers had no statistically significant difference in testing. Russian speakers were statistically significantly more likely than EP patients to have an X-ray/U/S (1.34, CI 1.14-1.59), EKG (1.26, CI 1.02-1.56), and stress test (1.80, CI 1.27-2.53). Spanish speakers were less likely to be admitted than EP patients (OR 0.8, CI 0.70-
0.91). Chinese, Cape Verdean, and Russian speakers had no statistically significant difference in rates of admission, but those speaking other languages had a statistically significant higher adjusted rate of admission (OR 1.35, CI 1.17-1.57).

\section{DISCUSSION}

In this large study of emergency department experiences for patients with limited English proficiency, we find that patients with limited English proficiency received more intensive care experiences. Although these findings reached statistically significance, they are small in absolute magnitude and it is not clear how clinically significant they are. Overall, LEP patients were more likely to receive Xray/U/S and more likely to be admitted to the hospital. Stratified analyses examining patients presenting with specific diagnoses related to the circulatory system showed increased use of stress tests, which was confirmed in sensitivity analyses. Basic imaging tests were higher in those with gastrointestinal diagnoses. Our findings were not uniform across all languages, which suggests that additional

Table 3 Comparisons of Outcomes by Discharge Diagnosis

\begin{tabular}{|c|c|c|c|c|c|c|c|}
\hline & \multicolumn{3}{|l|}{ Unadjusted percentage $\dagger$} & \multicolumn{2}{|c|}{ Unadjusted OR } & \multicolumn{2}{|c|}{ Adjusted OR } \\
\hline & Limited English proficient & English proficient & $p$ value* & OR & $95 \% \mathrm{CI}$ & OR & $95 \% \mathrm{CI}$ \\
\hline \multicolumn{8}{|c|}{$\begin{array}{l}\text { Circulatory system } \\
n=8497\end{array}$} \\
\hline EKG & 93.6 & 91.9 & .08 & 1.28 & $0.96-1.71$ & 1.26 & $0.91-1.74$ \\
\hline Stress test & 20.1 & 15.1 & .0001 & 1.41 & $1.18-1.69$ & 1.51 & $1.22-1.86$ \\
\hline Admission & 53.8 & 50.8 & .09 & 1.12 & $0.97-1.30$ & 0.78 & $0.65-0.94$ \\
\hline \multicolumn{8}{|l|}{$\begin{array}{l}\text { Digestive } \\
n=4872\end{array}$} \\
\hline $\mathrm{X}$-ray/U/S & 32.8 & 26.3 & .004 & 1.37 & $1.10-1.70$ & 1.31 & $1.02-1.68$ \\
\hline CT/MRI & 36.0 & 34.3 & .47 & 1.08 & $0.87-1.33$ & 1.07 & $0.84-1.37$ \\
\hline Admission & 63.4 & 63.4 & .66 & 1.04 & $0.84-1.29$ & 1.16 & $0.89-1.50$ \\
\hline
\end{tabular}

CT computed tomography, CI confidence interval, EKG electrocardiogram, MRI magnetic resonance imaging, OR odds ratio, U/S ultrasound * Comparisons with $p<.05$

†resented as percentage of total of LEP or EP population

$\ddagger$ Odds ratios with EP as reference. Models are adjusted for age, sex, race, marital status education, insurance type, time of presentation, and triage score 
factors related to culture also influence these care patterns. Not surprisingly, given higher rates of admission, there was no difference in 72-h unscheduled revisits.

Prior studies conducted in children have shown that patients with LEP were more likely to receive diagnostic testing in both the ED and inpatient setting. ${ }^{29}, 30$ The few studies in the adult population, however, have demonstrated variable findings. One prior study of adult patients in the ED did not find a difference in testing for those presenting with chest pain but did find a higher rate of abdominal CT scans in LEP patients presenting with abdominal pain. That study, however, was limited to those two diagnoses and had a sample size of just 324 patients. Another small study of 500 patients limited to four languages in an adult ED presenting with complaints of chest pain, shortness of breath, abdominal pain, headache, and pelvic pain found that LEP patients received fewer tests than EP patients even when an interpreter was present. ${ }^{24}$ Another study looking exclusively at Spanish speaking patients with ICD-9 codes related to abdominal pain and chest pain found that easy access to telephonic interpreters did not alter the rate of diagnostic testing or admission in Spanish speakers compared to English speakers. ${ }^{25}$ Our study is unique in not only its size but also our inclusion of the range of languages and discharge diagnoses seen in the ED. We also performed a sensitivity analysis of those with chief complaints related to chest pain or abdominal pain, rather than relying solely on diagnosis code, which is assigned after all testing is complete. These analyses, which focus attention on the time in the clinical encounter with the greatest diagnostic uncertainty and possibly the point at which language barriers may most impact the trajectory of care, showed consistent results.

We found that differences in admissions were driven primarily by the least frequently encountered languages. One potential explanation for this may be use of interpreter services in the care of patients in the emergency setting. The ED we studied has Spanish, Russian, Cantonese/Mandarin, and Cape Verdean/ Portuguese interpreters available around the clock, either in person or by telephone, though in person interpretation occurs mainly in the daytime. Interpretation for "other languages" more often requires contacting a contracted interpreter service via the phone. Prior studies have demonstrated that interpreters are only used in about one quarter of clinical encounters in the ED, which may exacerbate communication barriers in this setting. ${ }^{18}, 20$ The limited research on professional interpreters in the ED demonstrates variable impact on rates of return visits, intensity of diagnostic testing, and provision of high value care in this setting. ${ }^{18,24,25,29}$ We note that our results were not fully consistent as we did see increased testing in both Spanish and Russian patients for whom interpreters are more readily available. Unfortunately, we lacked data on whether interpreters were used during the clinical encounters included in our study.

The variable results observed after stratifying by language also suggest that care differences for LEP patients likely reflect more than failures in communication - a reminder that LEP patients are not a homogenous group. LEP individuals come from different cultures and thus have different expectations, beliefs, and experiences related to health care. Such differences in culture likely exacerbate communication failures. Not only language but also the words patients use to describe illness may differ. Consequently, translation may not be sufficient to reduce differences in care. Rather, linguistic interpretation and better cultural understanding may be necessary to provide truly equitable care.

As the US health care system shifts its orientation from volume to value, identifying sources of potential savings in the health care system become important. After controlling for acuity and discharge diagnosis, our findings of increased rates of admissions present an opportunity to understand why these differences exist and if they represent low-value care. If these patients might not have required hospital care, it becomes paramount that efforts be made to improve communication with LEP patients in the ED, whether through better availability of interpreter services, increased use of staff who speak the languages of presenting patients, or improved cultural competency. If efforts are made to reduce potentially unnecessary admissions, however, close attention will need to be paid to rates of unscheduled return visits. In contrast to our findings, some prior research has shown that LEP patients had higher rates of 72-h unscheduled return visits. ${ }^{19}, 20$

There are several limitations to our study. First, this was a single-center study, and therefore, the results may not be generalizable to other institutions. We did, however, study over 50,000 visits to a large tertiary center that was also a level 1 trauma center. Second, we defined LEP based on language preference given by patients at the time of first encounter with the hospital. Thus, although these data were universally available, some patients might have been reasonably proficient in English despite expressing a preference for a different language. Third, our measure of acuity, the triage score, might not have fully captured the level of acuity and might have been biased towards higher acuity scores for LEP patients related to uncertainty. ${ }^{31}$ Both of these limitation, however, would likely bias our results towards the null. Additionally, we were not able to account for patient's comorbidities. We also were unable to account for use of interpreter services during the visit, and use of interpreters may play an important role in mitigating the differences we observed. Although we did not have data on which visits had an interpreter present, we know that there are about 1700 face-to-face sessions with a live interpreter annually (with additional ones after hours by phone). However, care in the ED often takes place over several hours and evolves as the clinical picture changes based on patients' changing symptoms and new data. Even if an interpreter was present initially, they may not be present throughout the entirety of the visit. In fact, research demonstrates that providers make calculated decisions regarding interpreters and will use an interpreter only for certain parts of a clinical encounter. ${ }^{32}$ Finally, as noted above, differences in care for LEP patients are likely to be multifactorial, and it therefore is difficult to ascertain the impact of language as opposed to culture to explain our outcomes. 
Table 4 Comparisons of Outcomes by Language

\begin{tabular}{|c|c|c|c|c|c|c|c|}
\hline & \multicolumn{3}{|l|}{ Unadjusted Percentage $\dagger$} & \multicolumn{2}{|c|}{ Unadjusted OR } & \multicolumn{2}{|c|}{ Adjusted OR $\leftarrow$} \\
\hline & Limited English proficient & English proficient & $p$ value* & OR & $95 \% \mathrm{CI}$ & OR & $95 \% \mathrm{CI}$ \\
\hline \multicolumn{8}{|l|}{ Spanish } \\
\hline X-ray/U/S & 42.3 & 38.1 & .0001 & 1.19 & $1.08-1.30$ & 1.20 & $1.08-1.34$ \\
\hline CT/MRI & 28.5 & 27.3 & 0.22 & 1.06 & $0.96-1.17$ & 1.43 & $1.34-1.54$ \\
\hline EKG & 49.3 & 45.7 & .0013 & 1.15 & $1.05-1.26$ & 1.07 & $0.94-1.21$ \\
\hline Stress test & 4.6 & 2.9 & $<.0001$ & 1.56 & $1.26-1.93$ & 1.65 & $1.24-2.21$ \\
\hline Admission & 32.9 & 34.4 & 0.18 & 0.93 & $0.85-1.03$ & 0.80 & $0.70-0.91$ \\
\hline \multicolumn{8}{|l|}{ Chinese } \\
\hline X-ray/U/S & 36.4 & 38.2 & .33 & 0.93 & $0.80-1.07$ & 1.00 & $0.84-1.18$ \\
\hline CT/MRI & 32.7 & 27.3 & .0009 & 1.29 & $1.11-1.50$ & 0.96 & $0.81-1.15$ \\
\hline EKG & 48.8 & 45.7 & .09 & 1.13 & $0.98-1.30$ & 0.85 & $0.70-1.03$ \\
\hline Stress test & 3.6 & 2.9 & .36 & 1.19 & $0.81-1.76$ & 1.47 & $0.93-2.32$ \\
\hline Admission & 39.7 & 34.4 & .0023 & 1.25 & $1.08-1.45$ & 0.86 & $0.71-1.03$ \\
\hline \multicolumn{8}{|l|}{$\begin{array}{l}\text { Cape Verdean } \\
n=662\end{array}$} \\
\hline X-ray/U/S & 40.9 & 38.1 & .13 & 1.12 & $0.96-1.31$ & 0.96 & $0.80-1.14$ \\
\hline CT/MRI & 28.8 & 27.3 & .37 & 1.08 & $0.91-1.28$ & 1.00 & $0.83-1.21$ \\
\hline EKG & 49.4 & 45.7 & .06 & 1.16 & $0.99-1.35$ & 0.83 & $0.68-1.02$ \\
\hline Stress test & 4.4 & 2.9 & .03 & 1.49 & $1.02-2.17$ & 1.19 & $0.77-1.83$ \\
\hline Admission & 29.9 & 34.4 & .01 & 0.81 & $0.68-0.96$ & 0.88 & $0.72-1.09$ \\
\hline \multicolumn{8}{|l|}{$\begin{array}{l}\text { Russian } \\
n=627\end{array}$} \\
\hline X-ray/U/S & 49.1 & 38.1 & $<.0001$ & 1.56 & $1.34-1.83$ & 1.34 & $1.14-1.59$ \\
\hline CT/MRI & 35.9 & 27.3 & $<.0001$ & 1.49 & $1.36-1.75$ & 0.99 & $0.84-1.18$ \\
\hline EKG & 74.2 & 45.7 & $<.0001$ & 3.40 & $2.84-4.07$ & 1.21 & $0.98-1.49$ \\
\hline Stress test & 6.7 & 2.9 & $<.0001$ & 2.39 & $1.75-3.28$ & 1.80 & $1.27-2.53$ \\
\hline Admission & 53.9 & 34.4 & $<.0001$ & 2.23 & $1.90-2.61$ & 0.84 & $0.70-1.00$ \\
\hline
\end{tabular}

CT computed tomography, CI confidence interval, EKG electrocardiogram, MRI magnetic resonance imaging, OR odds ratio, U/S ultrasound *Comparisons with $p<.05$

$\uparrow$ Presented as percentage of total of LEP or EP population

$\neq$ Odds ratios with EP as reference. Models are adjusted for age, sex, race, marital status education, insurance type, time of presentation, triage score, and discharge diagnosis

In conclusion, we find that ED patients with limited English proficiency experienced statistically significant increased rates of certain diagnostic testing and of hospital admission, though these differences may not have uniform clinical significance. Further studies are needed to examine why these differences occurred and if they represent inefficiencies in care. More research also is needed on strategies to reduce disparities in care in the ED setting including increased use of trained interpreters and cultural competency training.

Acknowledgements: The authors would like to thank InSIGHT Core in the Center for Healthcare Delivery Science for assistance in obtaining primary data and programming assistance.

Corresponding Author: Bruce E. Landon, M.D., M.B.A., M.Sc.; Department of Health Care Policy Harvard Medical School, Boston, MA, USA (e-mail: landon@hcp.harvard.edu).

\section{Compliance with Ethical Standards:}

Conflict of Interest: The authors declare that they do not have conflicts of interest.

\section{REFERENCES}

1. Boissy A, Windover AK, Bokar D, et al. Communication Skills Training for Physicians Improves Patient Satisfaction. $J$ Gen Intern Med. 2016;31(7):755-761. doi:https://doi.org/10.1007/s11606-016-3597-2
2. Johnson MB, Castillo EM, Harley J, Guss DA. Impact of Patient and Family Communication in a Pediatric Emergency Department on Likelihood to Recommend: Pediatr Emerg Care. 2012;28(3):243-246. doi:https://doi.org/10.1097/PEC.0b013e3182494c83

3. Mira JJ, Orozco-Beltrán D, Pérez-Jover V, et al. Physician patient communication failure facilitates medication errors in older polymedicated patients with multiple comorbidities. Fam Pract. 2013;30(1):56-63. doi:https://doi.org/10.1093/fampra/cms046

4. Camille Ryan. Language Use in the United States:2011. American Community Survey Reports. U S Census Bur. http://www.census.gov/ prod/2013pubs/acs-22.pdf?cssp=SERP.

5. DuBard CA, Gizlice $\mathbf{Z}$. Language spoken and differences in health status, access to care, and receipt of preventive services among US Hispanics. Am J Public Health. 2008;98(11):2021-2028. doi:https://doi.org/10. 2105/AJPH.2007.119008

6. Fernandez A, Schillinger D, Warton EM, et al. Language barriers, physician-patient language concordance, and glycemic control among insured Latinos with diabetes: the Diabetes Study of Northern California (DISTANCE). J Gen Intern Med. 2011;26(2):170-176. doi:https://doi.org/ 10.1007/s11606-010-1507-6

7. Karliner LS, Kim SE, Meltzer DO, Auerbach AD. Influence of language barriers on outcomes of hospital care for general medicine inpatients. J Hosp Med. 2010;5(5):276-282. doi:https://doi. org/10.1002/jhm.658

8. Rodriguez F, Hong $\mathbf{C}$, Chang $\mathbf{Y}$, et al. Limited English proficient patients and time spent in therapeutic range in a warfarin anticoagulation clinic. $J$ Am Heart Assoc. 2013;2(4):e000170. doi:https://doi.org/10.1161/JAHA. 113.000170

9. Wisnivesky JP, Krauskopf $\mathbf{K}$, Wolf $\mathbf{M S}$, et al. The association between language proficiency and outcomes of elderly patients with asthma. Ann Allergy Asthma Immunol Off Publ Am Coll Allergy Asthma Immunol. 2012;109(3):179-184. doi:https://doi.org/10.1016/j.anai.2012.06.016

10. Divi C, Koss RG, Schmaltz SP, Loeb JM. Language proficiency and adverse events in US hospitals: a pilot study. Int $J$ Qual Health Care $J$ Int Soc Qual Health Care. 2007;19(2):60-67. doi:https://doi.org/10.1093/ intqhe/mzl069 
11. John-Baptiste A, Naglie G, Tomlinson G, et al. The effect of English language proficiency on length of stay and in-hospital mortality. $J$ Gen Intern Med. 2004;19(3):221-228.

12. Kim EJ, Kim T, Paasche-Orlow MK, Rose AJ, Hanchate AD. Disparities in Hypertension Associated with Limited English Proficiency. J Gen Intern Med. 2017;32(6):632-639. doi:https://doi.org/10.1007/s11606-0173999-9

13. Leung LB, Vargas-Bustamante A, Martinez AE, Chen X, Rodriguez HP. Disparities in Diabetes Care Quality by English Language Preference in Community Health Centers. Health Serv Res. October 2016. doi:https:// doi.org/10.1111/1475-6773.12590

14. Hahn EA, Burns $\mathbf{J}$, Jacobs EA, et al. Health Literacy and PatientReported Outcomes: A Cross-Sectional Study of Underserved Englishand Spanish-Speaking Patients With Type 2 Diabetes. J Health Commun. 2015;20(sup2):4-15. doi:https://doi.org/10.1080/10810730.2015. 1061071

15. Arthur KC, Mangione-Smith R, Meischke H, et al. Impact of English proficiency on care experiences in a pediatric emergency department. Acad Pediatr. 2015;15(2):218-224. doi:https://doi.org/10.1016/j.acap. 2014.06.019

16. Karliner LS, Auerbach A, Nápoles A, Schillinger D, Nickleach D, Pérez-Stable EJ. Language Barriers and Understanding of Hospital Discharge Instructions: Med Care. 2012;50(4):283-289. doi:https://doi. org/10.1097/MLR.0b013e318249c949

17. Schenker Y, Pérez-Stable EJ, Nickleach D, Karliner LS. Patterns of interpreter use for hospitalized patients with limited English proficiency. $J$ Gen Intern Med. 2011;26(7):712-717. doi:https://doi.org/10.1007/ s11606-010-1619-Z

18. Ramirez D, Engel KG, Tang TS. Language interpreter utilization in the emergency department setting: a clinical review. $J$ Health Care Poor Underserved. 2008;19(2):352-362. doi:https://doi.org/10.1353/hpu.0. 0019

19. Gallagher RA, Porter S, Monuteaux MC, Stack AM. Unscheduled return visits to the emergency department: the impact of language. Pediatr Emerg Care. 2013;29(5):579-583. doi:https://doi.org/10.1097/ PEC.0b013e31828e62f4

20. Ngai KM, Grudzen CR, Lee R, Tong VY, Richardson LD, Fernandez A The Association Between Limited English Proficiency and Unplanned Emergency Department Revisit Within 72 Hours. Ann Emerg Med. 2016;68(2):213-221. doi:https://doi.org/10.1016/j.annemergmed. 2016.02.042

21. Johns Hopkins University. Improving the Emergency Department Discharge Process: Environment Scan Report. Rockville, MD: Agency for Healthcare Research and Quality; 2014.
22. Njeru JW, St Sauver JL, Jacobson DJ, et al. Emergency department and inpatient health care utilization among patients who require interpreter services. BMC Health Serv Res. 2015;15:214. doi:https://doi.org/10. 1186/s12913-015-0874-4

23. Waxman MA, Levitt MA. Are diagnostic testing and admission rates higher in non-English-speaking versus English-speaking patients in the emergency department? Ann Emerg Med. 2000;36(5):456-461. doi:https://doi.org/10.1067/mem.2000.108315

24. Bernstein J, Bernstein E, Dave A, et al. Trained medical interpreters in the emergency department: effects on services, subsequent charges, and follow-up. J Immigr Health. 2002;4(4):171-176. doi:https://doi.org/10. 1023/A: 1020125425820

25. Jacobs EA, Fu PC, Rathouz PJ. Does a Video-Interpreting Network Improve Delivery of Care in the Emergency Department? Health Serv Res. 2012;47(1pt2):509-522. doi:https://doi.org/10.1111/j.1475-6773.2011. 01329.x

26. Doupe MB, Palatnick W, Day S, et al. Frequent users of emergency departments: developing standard definitions and defining prominent risk factors. Ann Emerg Med. 2012;60(1):24-32. doi:https://doi.org/10. 1016/j.annemergmed.2011.11.036

27. Centers for Disease Control and Prevention. International Classification of Diseases, Ninth Revision, Clinical Modification (ICD-9-CM). 2013. http://www.cdc.gov/nchs/icd/icd9cm. htm.

28. Emergency Severity Index (ESI): A Triage Tool for Emergency Department. Rockville, MD: Agency for Healthcare Research and Quality; 2013. http://www.ahrq.gov/professionals/systems/hospital/esi/index.html.

29. Hampers LC, Cha S, Gutglass DJ, Binns HJ, Krug SE. Language barriers and resource utilization in a pediatric emergency department. Pediatrics. 1999;103(6 Pt 1):1253-1256.

30. Levas MN, Cowden JD, Dowd MD. Effects of the limited English proficiency of parents on hospital length of stay and home health care referral for their home health care-eligible children with infections. Arch Pediatr Adolesc Med. 2011;165(9):831-836. doi:https://doi.org/10. 1001/archpediatrics.2011.61

31. Rogers AJ, Delgado CA, Simon HK. The effect of limited English proficiency on admission rates from a pediatric ED: stratification by triage acuity. Am J Emerg Med. 2004;22(7):534-536.

32. Hsieh E. Not just "getting by": factors influencing providers' choice of interpreters. J Gen Intern Med. 2015;30(1):75-82. doi:https://doi.org/10. 1007/s11606-014-3066-8 